
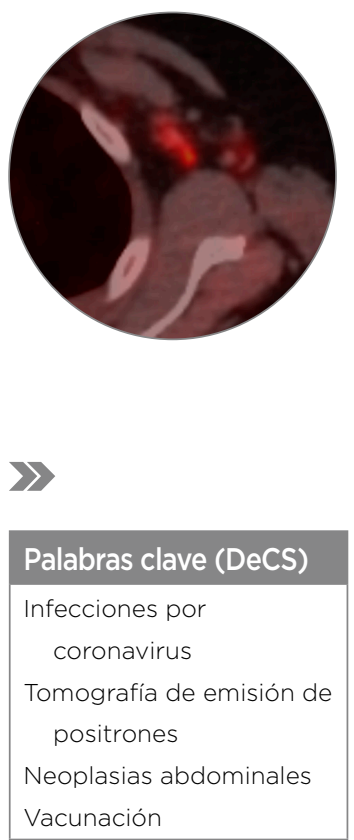

\section{Key words (MeSH)}

Coronavirus infections

Positron-emission

tomography

Abdominal neoplasms

Vaccination

\title{
Ganglios linfáticos axilares hipermetabólicos en PET-FDG posvacunación contra COVID-19, en pacientes con cáncer
}

\author{
Hypermetabolic axillary lymph nodes in PET-FDG post-vaccination \\ against COVID-19, in cancer patients
}

David Andrés Lizarazo Ortega' Patricia Bernal Trujillo² Gonzalo Ucrós Rodríguez ${ }^{2}$

\section{Resumen}

Al igual que las células malignas, las células inflamatorias pueden captar intensamente ${ }^{18} \mathrm{~F}$-fluorodesoxiglucosa (FDG). La reacción inflamatoria ganglionar axilar se ha descrito en forma creciente y globalmente luego de la vacunación contra COVID-19 con las vacunas de Pfizer, Moderna y AstraZeneca, en pacientes con cáncer. Se presentan dos casos con diagnóstico de cáncer de ovario y mama, respectivamente, con antecedente de vacunación reciente contra COVID-19, en quienes el estudio de PET/TC muestra ganglios linfáticos axilares hipermetabólicos con FDG. A continuación, se describen los hallazgos clave para interpretar el realce aumentado de los ganglios linfáticos axilares en pacientes con cáncer que han sido vacunados contra este virus.

\section{Summary}

Like malignant cells, inflammatory cells can intensely take up F18-fluorodeoxyglucose (FDG). The axillary lymph node inflammatory reaction has been increasingly and globally described after vaccination against COVID-19 with the Pfizer, Moderna and AstraZeneca vaccines, in cancer patients. We present two cases with a diagnosis of ovarian and breast cancer respectively, with a history of recent vaccination against COVID-19, in whom the PET/CT study present hypermetabolic axillary lymph nodes with FDG. Key findings for interpreting increased axillary lymph node uptake in cancer patients who have been vaccinated against this virus are described below.

\section{Introducción}

La tomografía por emisión de positrones (PET) es de utilidad en el diagnóstico y seguimiento de pacientes con enfermedades malignas. El ${ }^{18} \mathrm{~F}$-fluorodesoxiglucosa (FDG) es el trazador más comúnmente usado con este fin. Debido a la naturaleza del metabolismo de la glucosa, los hallazgos de ${ }^{18}$ F-FDG-PET/TC no son específicos para cáncer y se pueden encontrar en lesiones inflamatorias o infecciosas, lo cual lleva a hallazgos falsos positivos en pacientes con tumores malignos (1).

Si bien es cierto que los casos de falsos positivos posvacunación antiviral se conocen desde hace varios años, asociados a la vacuna contra la influenza (2) -incluyendo contra H1N1 (3)-, la pandemia por COVID-19 ha llevado a la vacunación masiva contra el virus SARS-CoV-2 y con ello a la aparición de casos de ganglios linfáticos axilares falsos positivos en pacientes con cáncer y otras patologías no malignas (4).

A continuación, se presentan dos casos de adenopatías axilares inflamatorias luego de vacunación contra COVID-19 en pacientes con cáncer.

\section{Presentación de casos}

\section{Caso 1}

Mujer de 49 años de edad con cáncer de ovario. ${ }^{18}$ F-FDG-PET/TC once días después de la aplicación de la vacuna contra COVID-19 en el miembro superior izquierdo (figura 1).

\section{Caso 2}

Mujer de 60 años de edad con cáncer de mama derecha. ${ }^{18} \mathrm{~F}$-FDG-PET/TC tres días después de la aplicación de la vacuna contra COVID-19 en el miembro superior izquierdo (figura 2).

\section{Discusión}

La reacción inflamatoria ganglionar axilar se ha descrito en forma creciente y global luego de la vacunación contra COVID-19 con las vacunas de Pfizer, Moderna y AstraZeneca, en pacientes con cáncer (seno, melanoma, linfoma, mieloma, cuello uterino, próstata, osteosarcoma y carcinoide) o con patologías no malignas (cardio\footnotetext{
Colombia.

e Imágenes Diagnósticas. Universidad El Bosque. FunBogotá, Colombia.

Especialista en Medicina
} 

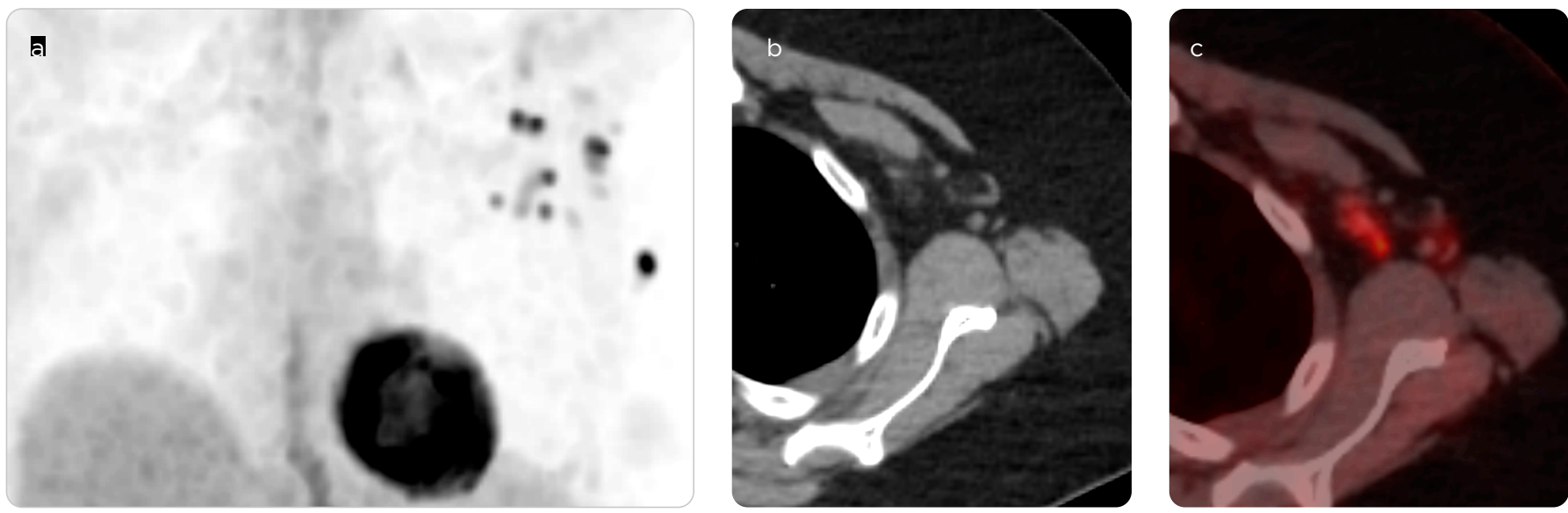

Figura 1. 18F-FDG-PET/TC. a) Proyección de máxima intensidad (MIP) coronal, b) TC axial de la región axilar y c) fusión PET/TC axial. Aumento de la captación de FDG en los ganglios axilares, que tienen forma ovalada con hilio graso central, de aspecto reactivo.
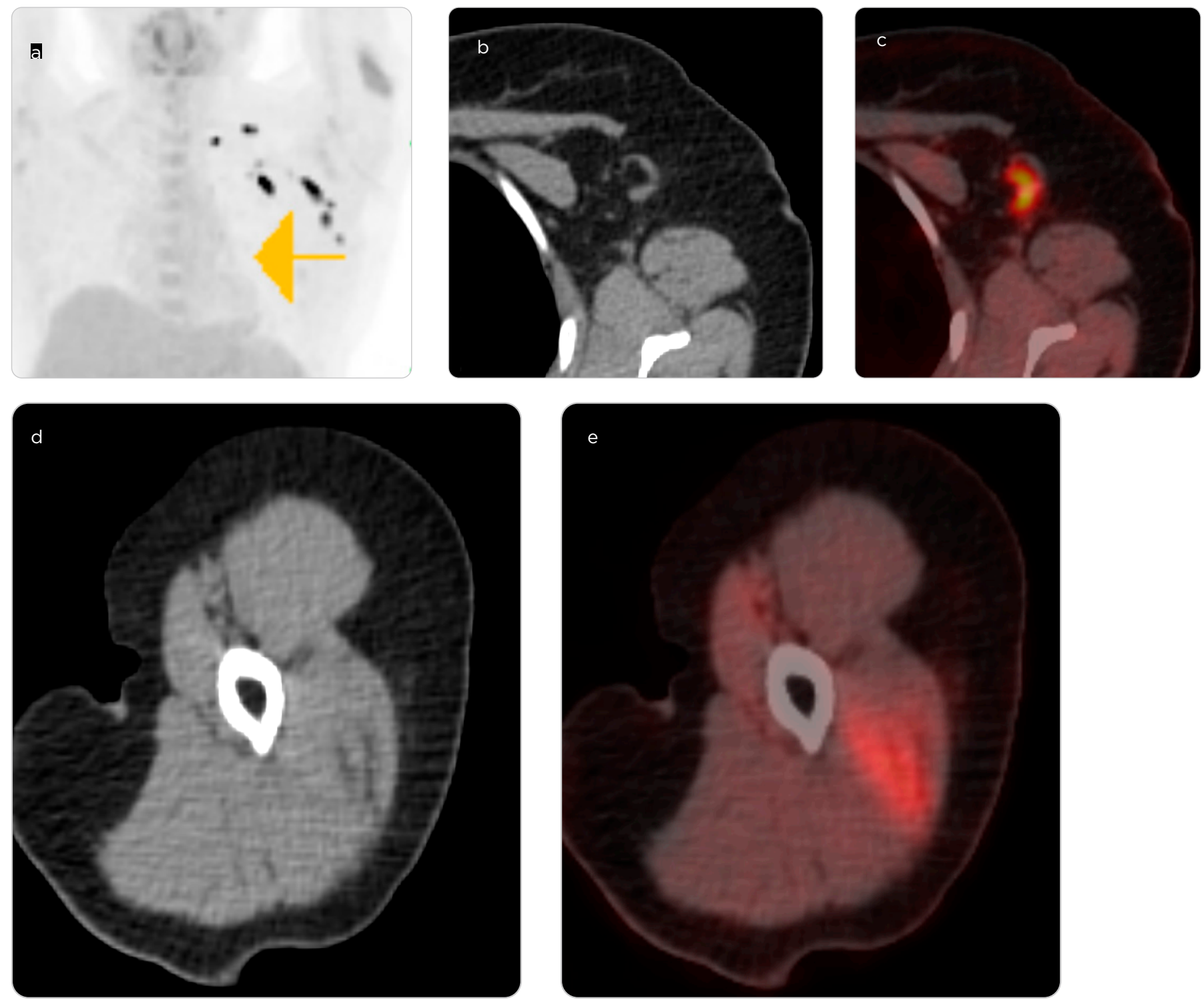

Figura 2. ${ }^{18} \mathrm{~F}-\mathrm{FDG}-\mathrm{PET} / \mathrm{TC}$. a) Proyección de máxima intensidad (MIP) coronal, b), TC axial región axilar, c) fusión PET/TC axial, d) TC axial región deltoidea y e) fusión PET/TC axial. Aumento de la captación de FDG en los ganglios axilares reactivos izquierdos y deltoides ipsilateral. 
vascular y sarcoidosis). Casi todos los estudios realizados fueron con ${ }^{18} \mathrm{~F}-\mathrm{FDG}-\mathrm{PET} / \mathrm{TC}$; en un caso se hizo PET/RM con el mismo trazador (5). Al igual que las células malignas, las células inflamatorias pueden captar intensamente el FDG debido al aumento del metabolismo de glucosa. Por ello, el FDG no es un marcador específico para las células malignas; a su vez, los ganglios linfáticos inflamatorios pueden captar y simular lesiones neoplásicas en la PET. De hecho, esta característica ha permitido su uso en el diagnóstico de enfermedades inflamatorias e infecciosas con buena sensibilidad diagnóstica (6).

Las anormalidades funcionales en los ganglios linfáticos demostradas por el aumento de la captación del FDG pueden preceder a las alteraciones morfológicas y por ello se pueden observar ganglios de tamaño normal reactivos (6), hallazgo señalado en los casos falsos positivos posvacunación (3).

Los hallazgos clave para interpretar la captación aumentada de los ganglios linfáticos axilares como un falso positivo en un paciente con cáncer y con antecedente de vacunación contra COVID-19 son los siguientes: aumento de la actividad del FDG en el músculo deltoides ipsilateral, los ganglios afectados son de tamaño normal o ligeramente aumentados $(9,3 \pm 1,7 \mathrm{~mm})$ y el antecedente de vacunación reciente, usualmente menos de 14 días $(1,7)$. Se ha descrito también captación en ganglios axilares del miembro superior en el que se aplicó vacuna contra COVID-19 en un paciente con sospecha de tumor neuroendocrino a quien se le practicó PET/TC con ${ }^{68} \mathrm{Ga}$-DOTA-TATE (4), que es un análogo radiomarcado de la somatostatina; el mecanismo de captación en este caso es diferente al del FDG y se explica porque los ganglios linfáticos también expresan receptores de somatostatina (5). Por lo anterior, en el estudio de PET/TC con ${ }^{68} \mathrm{Ga}$-DOTA-TATE también se pueden observar ganglios reactivos inflamatorios (5).

Los casos presentados deben alertar al especialista en imágenes diagnósticas sobre la posibilidad de este falso positivo. Por consiguiente, resulta esencial saber interpretar la captación aumentada de los ganglios linfáticos axilares, con el fin de no confundirlos con patología maligna.

Además, de ser posible, se recomienda la realización de este estudio de imagen en el seguimiento de pacientes con cáncer después de dos semanas de la vacunación contra COVID-19. Estos hallazgos en COVID-19 tienden a resolverse después de 12-14 días, pero pueden persistir hasta por 4 a 6 semanas (8).

\section{Referencias}

1. Burger IA, Husmann L, Hany TF, Schmid DT, Schaefer NG. Incidence and intensity of F-18 FDG uptake after vaccination with H1N1 vaccine. Clin Nucl Med. 2011;36(10):848-53.

2. Williams G, Joyce RM, Parker JA. False-positive axillary lymph node on FDG-PET/ CT scan resulting from immunization. Clin Nucl Med. 2006;31(11):731-2.

3. Panagiotidis E, Exarhos D, Housianakou I, Bournazos A, Datseris I. FDG uptake in axillary lymph nodes after vaccination against pandemic (H1N1). Eur Radiol. 2010;20(5):1251-3.

4. Treglia G, Cuzzocrea M, Muoio B, Elzi L. PET findings after COVID-19 vaccination: "Keep Calm and Carry On". Clin Transl Imaging. 2021;9(3):1-6.

5. Weiler-Sagie M, Half E. [68Ga]Ga-DOTA-TATE uptake due to COVID-19 vaccination. Eur J Nucl Med Mol Imaging. 2021:1-2.

6. Treglia G. Diagnostic performance of $18 \mathrm{~F}-\mathrm{FDG}$ PET/CT in infectious and inflammatory diseases according to published meta-analyses. Contrast Media Mol Imaging. 2019;2019:3018349.

7. González-Gómez S, Lizarazo DA, Romero J. FDG PET/CT scan after vaccination in times of pandemic. Radiology. 2021;299(3):E286. doi: http://dx.doi.org/10.
8. McIntosh LJ, Bankier AA, Vijayaraghavan GR, Licho R, Rosen MP. COVID-19 vaccination-related uptake on FDG PET/CT: An emerging dilemma and suggestions for management. AJR Am J Roentgenol. 2021;217(4):975-83.

\section{Correspondencia}

David Andrés Lizarazo Ortega

Calle 120 \# 7-33

Bogotá, Colombia

davidlizarazo_93@hotmail.com

Recibido para evaluación: 10 de enero de 2021

Aceptado para publicación: 17 de febrero de 2021 